Research Paper

\title{
Contrasting Cellularity and Fatty Acid Composition in Fat Depots from Alentejana and Barrosã Bovine Breeds Fed High and Low Forage Diets
}

\author{
Ana S.H. Costa ${ }^{1 \star}$, Paula A. Lopes ${ }^{1 \star}$, Marta Estevão1, Susana V. Martins ${ }^{1}$, Susana P. Alves ${ }^{2,3}$, Rui M.A. Pin- \\ to ${ }^{4}$, Hugo Pissarra1, Jorge J. Correia ${ }^{1}$, Mário Pinho' ${ }^{1}$ Carlos M.G.A. Fontes ${ }^{1}$, José A.M. Prates ${ }^{1 凶}$ \\ 1. CIISA, Faculdade de Medicina Veterinária, Universidade Técnica de Lisboa, Portugal. \\ 2. Unidade de Produção Animal, L-INIA, INRB, I.P., Vale de Santarém, Portugal. \\ 3. REQUIMTE, Instituto de Ciências Biomédicas de Abel Salazar, Universidade do Porto, Vairão VC, Portugal. \\ 4. iMed.UL, Faculdade de Farmácia, Universidade de Lisboa, Portugal. \\ * Authors who contributed equally.
}

$\triangle$ Corresponding author: José A. M. Prates, Faculdade de Medicina Veterinária, Av. da Universidade Técnica, Pólo Universitário do Alto da Ajuda, 1300-477 Lisboa, Portugal. Telephone: (+) 351 213652890; Fax: (+) 351 213652895. E-mail: japrates@fmv.utl.pt

() Ivyspring International Publisher. This is an open-access article distributed under the terms of the Creative Commons License (http://creativecommons.org/ licenses/by-nc-nd/3.0/). Reproduction is permitted for personal, noncommercial use, provided that the article is in whole, unmodified, and properly cited.

Received: 2011.07.28; Accepted: 2011.10.31; Published: 2012.01.01

\begin{abstract}
During the finishing phase of bovines, large amounts of subcutaneous and visceral fats are deposited leading to production inefficiencies with major impact on meat quality. A better understanding of the cellularity features of the main fat depots could provide strategies for adipose tissue manipulation. This study assessed the effect of feeding diets with distinct forage to concentrate ratios on the cellularity of two fat depots of beef cattle and their implications on the fatty acid profile. Thus, two phylogenetically distant Portuguese bovine breeds, Alentejana and Barrosã, were selected. The results did not show differences in subcutaneous fat deposition nor in visceral fat depots partitioning. Plasma adipokines concentration failed to show a consistent relationship with fatness, as leptin remained constant in all experimental groups, whereas interleukin- 6 was influenced by breed. Fat depot seems to determine the area and number of adipocytes, with larger adipocytes and a lower number of cells in subcutaneous fat than in mesenteric fat. Neither breed nor diet influenced adipocytes area and number. The contents of total fatty acids, partial sums of fatty acids and conjugated linoleic acid isomeric profile were affected by breed and fat depot. The incorporation of saturated fatty acids (SFA), trans fatty acids, polyunsaturated fatty acids (PUFA) and branched chain fatty acids (BCFA) was higher in mesenteric fat depot, whereas subcutaneous fat depot had greater percentages of monounsaturated fatty acids (MUFA). In addition, SFA and MUFA proportions seem to be breed-related. In spite of the less relevant role of diet, the percentages of PUFA and BCFA were influenced by this factor. Under these experimental conditions, the effect of fat depot on cellularity and fatty acid composition prevails over breed or diet, as reinforced by the principal component analysis.
\end{abstract}

Key words: bovine, fat depots, cellularity, fatty acid composition, adipokines.

\section{Introduction}

The manipulation of fat deposition in beef cattle is of major importance for the improvement of production efficiency, carcass composition and meat quality. In fact, subcutaneous and visceral fat depots are often not appreciated and, therefore, considered as "waste fat", whereas intramuscular fat is valued and regarded as "taste fat" [1]. Thus, the development of strategies to manipulate adipose tissue deposition in 
farm animals has been one of the major breeding goals for many years [2].

White adipose tissue, formerly regarded as a passive lipid storage site, is now recognized as a dynamic tissue [3]. It participates in general metabolism by providing substrate for the energy-consuming processes of almost all tissues. The metabolic activity of adipocytes in bovines is under the influence of several factors, namely breed, diet and fat depot location [4]. In addition, adipocytes are connected to the vascular network and display an important endocrine role. As developing pre-adipocytes differentiate into mature adipocytes, they acquire the ability to secrete various proteins [5], collectively known as adipokines, like leptin and interleukin-6 (IL-6). Leptin is an offensive cytokine that controls food intake and energy expenditure, thus regulating feeding behavior [6]. It also mitigates insulin resistance by stimulating beta-oxidation of fatty acids in the skeletal muscle [6]. IL-6 has a pro-inflammatory activity associated with obesity, impaired glucose tolerance and insulin resistance [3].

Different metabolic properties, including the regulation of lipid deposition, have been reported in several species for adipocytes of distinct anatomical locations [7]. Fatty acids of adipocytes derive from de novo synthesis or from diet. In cattle, the finishing system can produce important changes in fat deposition, thus suggesting that enzymes involved in lipogenesis are sensitive to dietary energy level and, possibly, to energy source. In fact, fat deposition is determined by the balance between lipogenesis and lipolysis. Lipogenesis is a process stimulated by a high carbohydrate diet but inhibited by polyunsaturated fatty acids (PUFA) intake and fasting [8]. Apart from the amount of fat [9], the fatty acid composition, including conjugated linoleic acid (CLA) isomers, of adipose tissue lipids is affected by dietary regimens and breed [10].

There is a breed-related pattern of fat deposition during bovines' growth [11]. However, the information available on the effect of genetic background on adipose tissue cellularity and fatty acid composition is scarce. Thus, further studies in this field are needed. Genetic distances have been described for some Portuguese autochthonous bovine breeds, independently of their geographical location [12]. Alentejana is a large bovine breed [13] usually reared on a traditional semi-extensive production system in the Southern plains of Portugal [14]. It is the most important commercial Portuguese Protected Designation of Origin (PDO) beef [15]. In contrast, Barrosã is a small breed [13] typically reared on a traditional production system in the mountainous Norwest of
Portugal [16], being the most consumed PDO-veal in Portugal [15]. In addition, large differences in the lipid composition and nutritional quality of intramuscular fat from Alentejana [17] and Barrosã [16] bovine meats have been described by our research group.

This experiment was designed to study the effect of breed and diet on cellularity and fatty acid biosynthesis of subcutaneous and mesenteric fat depots from young bulls. For this purpose, two phylogenetically distant autochthonous bovine breeds (Alentejana and Barrosã) and two experimental diets (based on $30 / 70 \%$ and $70 / 30 \%$ of silage and concentrate, respectively) were selected. We hypothesized that: i) the genetic background can determine the bovine fat deposition and partitioning; ii) rearing cattle on different silage/concentrate ratios can alter the fatty acid composition of adipose tissues; iii) the lipid deposition may vary according to the fat depot considered. To achieve these aims, adipocytes size and number (per area) of subcutaneous and mesenteric fat depots were evaluated, through histometrical analysis, in parallel with plasma determination of some adipokines (leptin and IL-6). To further characterize these effects upon cellularity of subcutaneous and mesenteric fats, the detailed fatty acid composition, including the CLA isomeric profile, was determined in both fat depots.

\section{Material and Methods}

\section{Experimental design}

This trial was conducted under the guidelines for the care and use of experimental animals of Unidade de Produção Animal, L-INIA, INRB (Fonte Boa, Vale de Santarém, Portugal). Forty young bulls from Alentejana (large-framed breed) and Barrosã (small-framed breed), were assigned to high or low forage based diets (four experimental groups of 10 animals each). Diets were composed of $30 / 70 \%$ and $70 / 30 \%$ of maize silage and concentrate, respectively. The proximate and fatty acid composition of both experimental diets were recently published [18]. The animals were housed in eight adjacent pens, two pens per breed and diet. The initial age was $331 \pm 32$ days for Alentejana bulls (average weight of $266 \pm 10.5 \mathrm{~kg}$ ) and $267 \pm 10$ days for Barrosã bulls (average weight of $213 \pm 3.64 \mathrm{~kg}$ ). The experiment lasted from January to November 2009. One Alentejana bull from the high silage diet was removed from the study due to a limp.

One week prior to slaughter, blood samples were collected from the tail vein and centrifuged (3000 rpm for 15 minutes at room temperature) to harvest heparinized plasma. The plasma was analyzed for some biochemical parameters within 24 hours at a Clinical 
Chemistry Laboratory (Clínica Médica e Diagnóstico Dr. Joaquim Chaves, Algés, Portugal). All animals were slaughtered at 18 months-old, which is the commercial slaughter age for young bulls in Portugal, at the INRB experimental abattoir by exsanguination after stunning with a cartridge-fired captive bolt stunner. Mesenteric, omental and kidney knob and channel fat (KKCF) depots were excised and weighed. Subcutaneous adipose tissue was sampled and its amount was determined by dissection of the leg joint. The former has been suggested to be representative of the overall bovine carcass composition, at least in these particular breeds [19]. For histometrical analyses, samples from subcutaneous and mesenteric fat depots (approximately $100 \mathrm{mg}$ ) were fixed by immersion in $10 \%$ neutral buffered formalin (Merck, Darmstadt, Germany) for 24 hours and processed for paraffin (Microscopy Histosec, Merck) embedding. A second aliquot from each fat depot was vacuum packed and stored at $-20{ }^{\circ} \mathrm{C}$ until lipid extraction and determination of fatty acid composition and CLA isomeric profile.

\section{Plasma metabolites and adipokines determi- nation}

Triacylglycerols (GPO-PAP) and glucose (GOD-PAP) levels were determined in plasma through diagnostic test kits (Roche Diagnostics, Mannheim, Germany) using a Modular Hitachi Analytical System (Roche Diagnostics). Plasma insulin was quantified using a Bovine ELISA kit (Mercodia, Uppsala, Sweden), leptin through a Multi-Species RIA kit (Linco Research, Millipore, MO, USA) and IL-6 using a Bovine ELISA kit (Cusabio Biotech Co., Ltd, Wuhan, Hubei Province, China).

\section{Histometrical analysis}

Adipose tissue sections with $10 \mu \mathrm{m}$ thick were cut on a microtome (Leica, SM 2000R, Nussloch, Germany) from each of the paraffin-embedded specimens. Sections were stained with the classical hematoxylin (Bio-optica, Milan, Italy) and eosin procedure (Richard-Allan Scientific, Kalamazoo, MI, USA) to assess morphology under a light microscope (Olympus BX51 equipped with a DP11 microscope digital camera system, Olympus, Tokyo, Japan). For

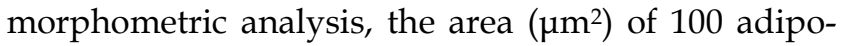
cytes from 5 fields per section was determined under the microscope (magnification of $\times 100$ ), using the DP software for image analysis (Olympus DP-Soft version 3.0 for Windows 95/98). The number of adipocytes was also determined in a fixed area of $\sim 560 \times 10^{3}$ $\mu \mathrm{m}^{2}$ per section (magnification of $\times 100$ ). The entire histological plan was followed as described by Corino et al. [20].

\section{Fatty acid composition}

Subcutaneous and mesenteric fat samples were lyophilised $\left(-60^{\circ} \mathrm{C}\right.$ and $\left.2.0 \mathrm{hPa}\right)$ and maintained at -20 ${ }^{\circ} \mathrm{C}$ until further analysis. Total lipids were extracted by the method of Folch et al. [21], using dichloromethane and methanol $(2: 1 \mathrm{v} / \mathrm{v})$ instead of chloroform and methanol $(2: 1 \mathrm{v} / \mathrm{v})$, as modified by Carlson [22]. Fatty acids were converted to methyl esters as described by Raes et al. [23], using sodium methoxide in anhydrous methanol $(0.5 \mathrm{~mol} / \mathrm{l})$ for $30 \mathrm{~min}$, followed by hydrochloric acid in methanol $(1: 1 \mathrm{v} / \mathrm{v})$ for $10 \mathrm{~min}$ at $50^{\circ} \mathrm{C}$. Fatty acid methyl esters (FAME) were extracted twice with $3 \mathrm{ml}$ of $n$-hexane and pooled extracts were evaporated at $35{ }^{\circ} \mathrm{C}$, under a stream of nitrogen, until a final volume of $2 \mathrm{ml}$. The resulting FAME were then analyzed by gas-liquid chromatography using a fused-silica capillary column (CP-Sil 88; $100 \mathrm{~m} \times 0.25 \mathrm{~mm}$ i.d., $0.20 \mathrm{~mm}$ film thickness; Chrompack, Varian Inc., Walnut Creek, CA, USA), equipped with a flame ionization detector, as described by Bessa et al. [24]. The quantification of FAME used nonadecanoic acid (19:0) as the internal standard, added to lipids prior to saponification and methylation. The same FAME solution was used for the analysis of both fatty acid composition and CLA isomeric profile, enabling the direct comparison of quantitative data and eliminating differences in sample preparation. CLA isomers were individually separated by triple silver-ion columns in series (ChromSpher 5 Lipids; $250 \mathrm{~mm} \times 4.6 \mathrm{~mm}$ i.d., $5 \mu \mathrm{m}$ particle size; Chrompack, Bridgewater, NJ, USA), using a high performance liquid chromatography (HPLC) system (Agilent 1100 Series, Agilent Technologies Inc., Palo Alto, CA, USA) equipped with an autosampler and a diode array detector adjusted to $233 \mathrm{~nm}$, according to the procedure previously reported [25]. The identification of individual CLA isomers was achieved by comparison of their retention times with commercial and prepared standards, as well as with values published in the literature. Fatty acid composition was expressed as g/100 g of total fatty acid content, assuming a direct relationship between peak area and fatty acid methyl ester weight. The amounts of CLA isomers were calculated from their $\mathrm{Ag}^{+}$-HPLC areas relative to the area of the main isomer cis(c)9, trans( $t$ )11 CLA identified by GC (which comprises both $t 7, c 9$ and $t 8, c 10$ CLA isomers), as described by Rego et al. [25].

\section{Statistics}

Values are presented as mean \pm standard error of the mean (SEM) for data concerning growth perfor- 
mance parameters, plasma metabolites and histological analysis. Data analysis was performed using the Statistical Analysis System (SAS) software package, v9.1 [26]. The effect of breed and diet as main factors, and their interaction (breed $\times$ diet), on the body composition and plasma biochemical parameters were analyzed by the General Linear Model to perform a two-way analysis of variance. Regarding the analysis of histometrical data, the Sturges' rule [27] was applied to define the number of classes. The analysis of variance on histometrical data and fatty acid profile was performed using the mixed model, considering the animal as a subject and the fat depot as repeated measures, because the two fats were collected from the same animal. Least squares means were determined using the LSMEANS option and compared, when significant (at $P<0.05$ ), using the probability difference procedure (PDIFF option). Pearson's correlation coefficients were applied to establish possible relationships between fat depots mass and their respective adipocytes area. The relationships between cellularity and fatty acid composition in both depots were assessed by the principal component analysis (PCA), using the PRINCOMP procedure of SAS. The PCA was applied to a data set of 78 samples and 36 variables to reduce the dimensionality of the data set and to describe the variability of data in two dimensions. The PCA was used to examine the relationship between the cellularity and fatty acid composition variables considered, enabling not only plots of the relationship between the variables but also attempting to explain those relationships. After data normalization, the principal components were considered significant if they contributed more than $5 \%$ for the total variance.

\section{Results}

\section{Fatness and diet are not clearly associated with plasma adipokines}

The overall characterization of the studied animal groups, concerning body composition parameters, plasma metabolites and adipokines, is presented in Table 1. Live slaughter weight was significantly influenced by breed $(P<0.001)$, being the values higher in Alentejana than in Barrosã bulls. Similarly, hot carcass and leg joint weights were higher in Alentejana relative to Barrosã bulls $(P<0.001)$. The dissection of the leg showed no differences among groups regarding the subcutaneous fat in the leg joint $(P>0.05)$. Mesenteric and omental fats, expressed relatively to the hot carcass weight, were higher in low silage fed animals $(P<0.05$ and $P<0.001$, respectively). The sum of perirenal and retroperitoneal fats (KKCF) showed no influence from breed or diet $(P>0.05)$.

Table I. Body composition parameters, plasma metabolites and adipokines from Alentejana and Barrosã bulls fed high (HS) or low (LS) silage diets.

\begin{tabular}{|c|c|c|c|c|c|c|c|c|}
\hline & \multicolumn{2}{|c|}{ Alentejana } & \multicolumn{2}{|c|}{ Barrosã } & \multirow[b]{2}{*}{ SEM } & \multicolumn{3}{|c|}{ Significance level } \\
\hline & HS & LS & HS & LS & & $\mathrm{B}$ & $\mathrm{D}$ & $\mathrm{B} \times \mathrm{D}$ \\
\hline \multicolumn{9}{|l|}{ Body composition parameters } \\
\hline Live slaughter weight (kg) & 622 & 636 & 457 & 497 & 22.3 & $* * *$ & ns & ns \\
\hline Hot carcass weight $(\mathrm{kg})$ & 357 & 371 & 257 & 284 & 13.1 & $* * *$ & ns & ns \\
\hline Leg joint weight $(\mathrm{kg})$ & 46.8 & 47.8 & 35.0 & 36.0 & 1.65 & $* * *$ & ns & ns \\
\hline Subcutaneous fat (g/100 g leg) & 4.10 & 4.59 & 4.54 & 5.92 & 0.459 & ns & ns & ns \\
\hline Mesenteric fat (g/kg carcass) & 15.5 & 16.8 & 15.2 & 20.9 & 1.44 & ns & * & ns \\
\hline Omental fat (g/kg carcass) & 21.1 & 24.1 & 19.0 & 28.4 & 1.65 & ns & $* * *$ & ns \\
\hline $\mathrm{KKCF}^{\mathrm{a}}$ (g/kg carcass) & 23.5 & 20.7 & 22.5 & 23.8 & 1.92 & ns & ns & ns \\
\hline \multicolumn{9}{|l|}{ Plasma metabolites and adipokines } \\
\hline Triacylglycerols (mg/L) & 175 & 176 & 170 & 184 & 15.8 & ns & ns & ns \\
\hline Glucose (mg/L) & 889 & 885 & 820 & 806 & 31.4 & * & ns & ns \\
\hline Insulin $(\mu \mathrm{g} / \mathrm{L})$ & 0.884 & 1.80 & 1.28 & 2.12 & 0.359 & ns & * & ns \\
\hline Leptin $(\mu \mathrm{g} / \mathrm{L})$ & 3.99 & 3.82 & 3.89 & 5.04 & 0.451 & ns & ns & ns \\
\hline Interleukin-6 (ng/L) & 11.2 & 8.88 & 18.4 & 17.8 & 3.21 & * & ns & ns \\
\hline
\end{tabular}

aKidney knob and channel fat. $\mathrm{B}=$ breed; $\mathrm{D}=$ diet. Significance level: not significant (ns), $P>0.05 ;{ }^{*}, P<0.05 ;{ }^{* *}, P<0.01 ;{ }^{* * *}, P<0.001$. 
Table 2. Effect of breed, diet and fat depot on the adipocytes area $\left(\mu \mathrm{m}^{2}\right)$, number (in $560 \times 10^{3} \mu \mathrm{m}^{2}$ ) and distribution of subcutaneous (Sub) and mesenteric (Mes) fats from Alentejana and Barrosã bulls fed high (HS) or low silage (LS) diets.

\begin{tabular}{|c|c|c|c|c|c|c|c|c|c|c|c|c|c|c|}
\hline & \multicolumn{4}{|c|}{ Alentejana } & \multicolumn{4}{|c|}{ Barrosã } & \multirow[b]{3}{*}{ SEM } & \multirow{2}{*}{\multicolumn{4}{|c|}{ Significance level }} & \multirow[b]{3}{*}{$\mathrm{B} \times \mathrm{D} \times \mathrm{FD}$} \\
\hline & \multicolumn{2}{|c|}{ HS } & \multicolumn{2}{|c|}{ LS } & \multicolumn{2}{|c|}{ HS } & \multicolumn{2}{|c|}{ LS } & & & & & & \\
\hline & Sub & Mes & Sub & Mes & Sub & Mes & Sub & Mes & & B & $\mathrm{D}$ & FD & $B \times D$ & \\
\hline Adipocytes & & & & & & & & & & & & & & \\
\hline Area & 6759 & 5353 & 5931 & 5217 & 6842 & 6087 & 7177 & 5676 & 466 & ns & ns & $* * *$ & ns & ns \\
\hline Number & 76.3 & 94.3 & 86.9 & 100 & 79.0 & 89.4 & 70.0 & 92.1 & 6.24 & ns & ns & $* * *$ & ns & ns \\
\hline Class $\left(\mu \mathrm{m}^{2}\right)$ & & & & & & & & & & & & & & \\
\hline $0-1800$ & $19.0^{\mathrm{a}}$ & $24.2^{\mathrm{abc}}$ & $29.2^{\mathrm{bc}}$ & $32.9 c$ & $20.1^{a}$ & $22.6^{\mathrm{ab}}$ & $21.6^{\mathrm{ab}}$ & $20.4^{\mathrm{a}}$ & 3.04 & * & * & ns & * & ns \\
\hline $1800-3600$ & 11.3 & 12.8 & 10.7 & 12.1 & 11.8 & 13.6 & 13.3 & 14.2 & 1.15 & ns & ns & ns & ns & ns \\
\hline $3600-5400$ & 12.5 & 14.9 & 11.2 & 11.5 & 11.6 & 12.0 & 10.1 & 13.8 & 1.12 & ns & ns & ns & ns & ns \\
\hline $5400-7200$ & $14.3^{\mathrm{abc}}$ & $16.2^{\mathrm{bc}}$ & $10.4^{\mathrm{a}}$ & $13.0^{\mathrm{ab}}$ & $11.6^{\mathrm{a}}$ & $13.4^{\mathrm{abc}}$ & $10.7^{a}$ & $17.4^{\mathrm{c}}$ & 1.58 & ns & ns & $* *$ & * & ns \\
\hline $7200-9000$ & $13.9^{\mathrm{ad}}$ & $11.8^{\mathrm{ac}}$ & $12.8^{\mathrm{abcd}}$ & $10.1^{\mathrm{c}}$ & $14.3^{\mathrm{ad}}$ & $10.4^{\mathrm{bc}}$ & $12.7 \mathrm{abc}$ & $14.9^{\mathrm{d}}$ & 1.14 & ns & ns & ns & ns & * \\
\hline $9000-10800$ & 9.17 & 10.2 & 9.31 & 7.56 & 10.3 & 10.3 & 8.58 & 9.87 & 1.09 & ns & ns & ns & ns & ns \\
\hline $10800-12600$ & 4.95 & 7.30 & 6.73 & 5.05 & 7.29 & 7.19 & 7.00 & 4.98 & 1.06 & ns & ns & $*$ & ns & ns \\
\hline $12600-14400$ & 5.40 & 2.27 & 5.54 & 4.25 & 7.70 & 3.66 & 6.44 & 2.97 & 0.954 & ns & ns & $* * *$ & ns & ns \\
\hline $14400-16200$ & 2.31 & 1.28 & 3.22 & 2.66 & 4.54 & 2.87 & 4.88 & 2.12 & 0.991 & * & ns & $*$ & ns & ns \\
\hline$\geq 16200$ & 4.87 & 1.50 & 3.62 & 3.51 & 4.24 & 4.31 & 8.21 & 3.06 & 1.90 & ns & ns & $*$ & ns & ns \\
\hline
\end{tabular}

$\mathrm{B}=$ breed; $\mathrm{D}=$ diet; $\mathrm{FD}=$ fat depot. $\mathrm{B} \times \mathrm{FD}$ and $\mathrm{D} \times \mathrm{FD}$ interactions were not significant $(P>0.05)$. Significance level: not significant (ns), $P>0.05$;

${ }^{*}, P<0.05 ;{ }^{* *}, P<0.01$; ${ }^{* * *}, P<0.001$; means in the same row with different superscripts are significantly different $(P<0.05)$.

No significant effects of breed, diet or interaction were observed for the content of plasma triacylglycerols $(P>0.05)$. The glucose levels in plasma were affected by breed.The values were higher in Alentejana compared to Barrosã bulls $(P<0.05)$. Insulin concentration was affected by diet $(P<0.05)$, as low silage diets fed to both breeds promoted higher values of this hormone. Plasma IL-6 concentration was affected by breed, with higher levels in Barrosã than in Alentejana bulls $(P<0.05)$. Leptin concentration in plasma was kept unchanged among the four experimental groups $(P>0.05)$.

\section{Fat depot, but neither breed nor diet, deter- mines cellularity}

Data concerning the histometrical evaluation of subcutaneous and mesenteric fats as affected by breed, diet and specific fat depot are summarized in Table 2. Except for the fat depot effect $(P<0.001)$, no other influence from breed, diet or their interaction was observed $(P>0.05)$ for adipocytes area and number. In fact, overall subcutaneous fat had larger adipocytes and a lower number of cells than mesenteric fat (6677 vs $5584 \mu^{2}$ and 78.0 vs 94.0 cells in $560 \times 10^{3}$ $\mu \mathrm{m}^{2}$, respectively).

Table 2 also displays data regarding adipocytes area distribution, according to breed, diet and fat depot. Based on the Sturges' rule, ten classes of adipocytes area were constructed. This analysis revealed that, for the smaller adipocytes, there were no differ- ences between fat depots $(P>0.05)$. The distribution of fat cells was significantly different in classes containing adipocytes ranging from 5400 to $7200 \mu \mathrm{m}^{2}$ and those larger than $10800 \mu \mathrm{m}^{2}$ (at least, $P<0.05$ ). Breed played a less relevant role on adipocytes filling and its effect was observed only for two classes. Alentejana bulls had a higher frequency of smaller adipocytes $\left(0-1800 \mu \mathrm{m}^{2}, P<0.05\right)$ and adipocytes ranging from 14400 to $16200 \mu \mathrm{m}^{2}(P<0.05)$.

The main finding of Table 2 is that subcutaneous and mesenteric fats were distinct in terms of adipocytes area and number. Following from this result, we decided to perform a statistical analysis for both fat depots separately (Figure 1). For the subcutaneous fat, a diet effect $(P<0.05)$ was observed in the class comprising medium adipocytes, ranging from $7200-9000$ $\mu \mathrm{m}^{2}$. Indeed, consistently lower frequencies of these medium adipocytes were observed in bulls fed on low silage diets. Moreover, the class with adipocytes ranging from 14400 to $16200 \mu \mathrm{m}^{2}$ was influenced by breed $(P<0.05)$ because the relative frequency of larger adipocytes was higher in Barrosã when compared to Alentejana bulls. In relation to the mesenteric fat, some interesting interactions were observed between breed and diet in the 3600-5400 $(P<0.05), 5400-7200$ $(P<0.05)$ and $7200-9000 \mu^{2} \quad(P<0.01) \quad$ classes. Alentejana bulls fed on low silage diet had lower frequencies of these medium adipocytes than their counterparts fed on high silage. The inverse trend was observed for Barrosã bulls. 

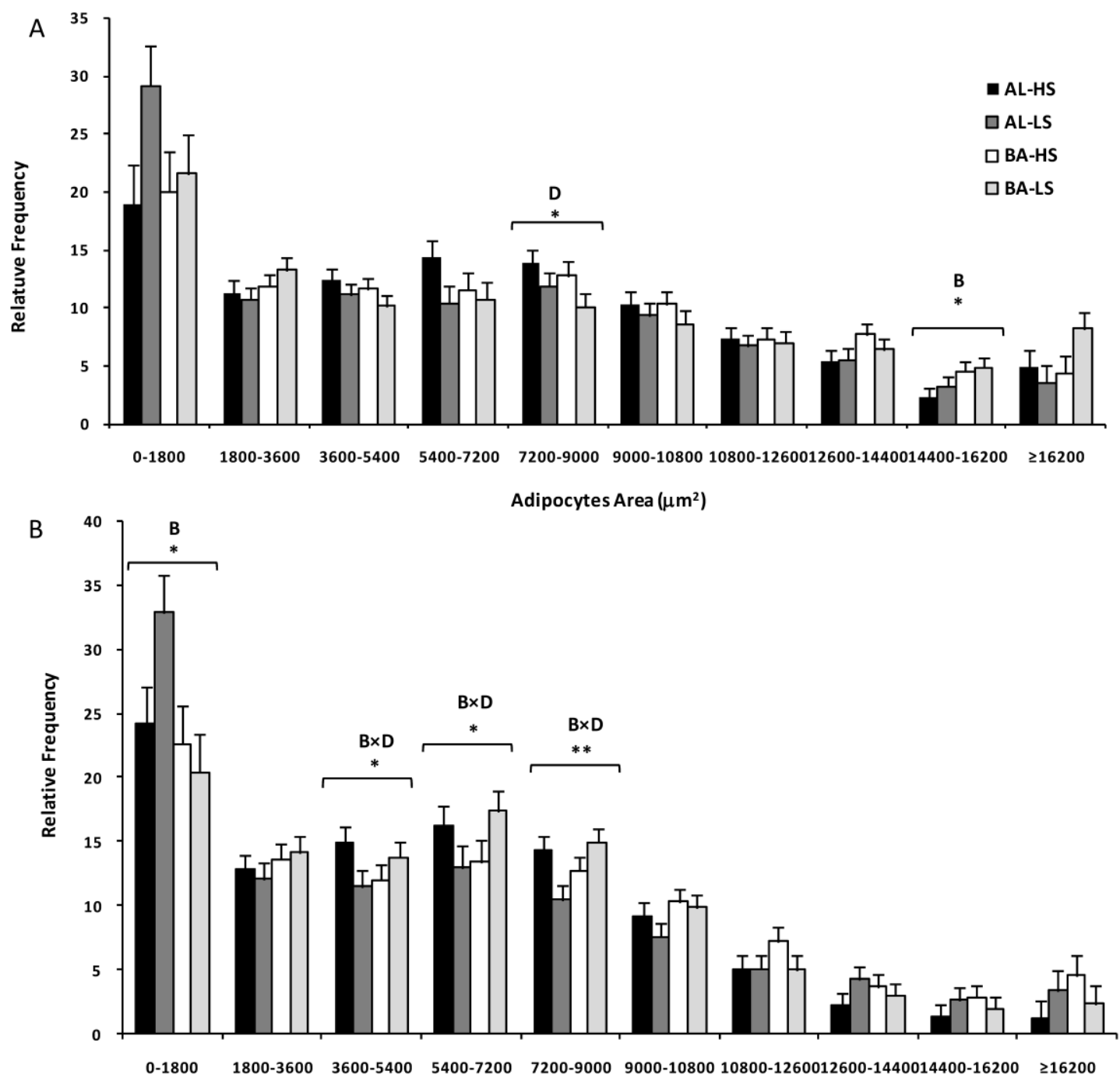

Adipocytes Area $\left(\mu \mathrm{m}^{2}\right)$

Figure I. Adipocytes area distribution of subcutaneous $(A)$ and mesenteric $(B)$ fats from Alentejana $(A L)$ and Barrosã $(B A)$ bulls fed high (HS) or low (LS) silage diets. $B=$ breed; $D=$ diet. Significance level: *, $P<0.05 ; * * P<0.0$ I.

The Pearson's correlation coefficient between adipose tissue depot weight and adipocytes area was significant for mesenteric fat $(r=0.36, P=0.023)$, in contrast to subcutaneous fat $(r=0.27, P=0.097)$.

\section{Breed and fat depot are major contributors to a contrasting fatty acid composition}

The fatty acid composition of both fat depots from the four experimental groups is depicted in Table 3 . The predominant saturated fatty acids (SFA) were palmitic (16:0) and stearic (18:0) acids, and oleic acid $(18: 1 c 9)$ was the main monounsaturated fatty acid (MUFA). While in the Alentejana breed the deposition of palmitic acid was higher in subcutaneous than in mesenteric fat depot, the opposite pattern was observed in the Barrosã breed, resulting in a breed $\times$ diet interaction $(P<0.001)$. Stearic and oleic fatty acids varied according to breed $(P<0.001)$ and fat depot $(P<0.001)$ considered. While stearic acid was higher in the mesenteric fat of Alentejana bulls, oleic acid was higher in the subcutaneous fat of the Barrosã breed. Trans octadecenoates (18:1t), regarded as the main intermediates arising from C18 PUFA ruminal biohydrogenation, varied according to the main factors. Both breed $(P<0.001)$ and fat depot $(P<0.001)$ determined the proportions of vaccenic acid (18:1t11), the main trans fatty acid (TFA). It was found a greater deposition of this fatty acid in the mesenteric fat from Barrosã breed in comparison to the other experimental groups. The main branched chain fatty acid (BCFA) found in the present study was $a-17: 0$, a microbial lipid, with the highest percentages observed in 
the subcutaneous fat of Barrosã bulls fed the high silage diet $(P<0.001)$. Linoleic acid $(18: 2 n-6)$ was the main PUFA, being determined by all main factors (at least, $P<0.05)$ and the interaction between breed and diet $(P<0.001)$. The highest proportions of linoleic acid were found in the mesenteric fat of Alentejana bulls fed the low silage diet. A similar pattern was found for $18: 3 n-3$, the second most abundant PUFA. Concerning the CLA isomeric profile, significant variations were observed among breeds, diets and fat depots (Table 4). The sum of CLA isomers was higher in the subcutaneous fat than in the mesenteric fat, as well as in the Barrosã breed relatively to the Alentejana breed $(P<0.001)$. Individual CLA isomers were mostly influenced by breed and fat depot (at least, $P<0.05)$, although the diet had a strong effect on $t 11, t 13, t 11, c 13$ and $t 7, c 9$ isomers $(P<0.001)$, amongst others. The high silage diet promoted greater proportions of $t 11, t 13, t 11, c 13$ and $t 12, t 14$, the latter reaching the highest value in the mesenteric fat of Alentejana breed. As far as the $110, c 12$ CLA isomer is concerned, a significant deposition was observed in Alentejana bulls fed on low silage diet, regardless the fat depot $(P<0.05)$. Not surprisingly, the predominant CLA isomer in both fat depots was the $c 9, t 11$, which was influenced by breed, diet and fat depot. Its highest value was observed in the subcutaneous fat of Barrosã bulls fed on high silage diet $(P<0.001)$. The proportions of the $t 7, c 9$ CLA isomer were also influenced by the main factors, that is, the highest values were observed in the subcutaneous fat of Alentejana bulls fed low silage diet $(P<0.001)$.

Table 3. Total fatty acid content ( $\mathrm{mg} / \mathrm{g}$ fat) and fatty acid composition ( $\mathrm{g} / \mathrm{l} 00 \mathrm{~g}$ fatty acids) of subcutaneous (Sub) and mesenteric (Mes) fats from Alentejana and Barrosã bulls fed high (HS) or low silage (LS) diets.

\begin{tabular}{|c|c|c|c|c|c|c|c|c|c|c|c|c|c|c|c|c|}
\hline & \multicolumn{4}{|c|}{ Alentejana } & \multicolumn{4}{|c|}{ Barrosã } & & & & & & & & \\
\hline & \multicolumn{2}{|c|}{ HS } & \multicolumn{2}{|c|}{ LS } & \multicolumn{2}{|c|}{ HS } & \multicolumn{2}{|c|}{ LS } & \multirow[b]{2}{*}{ SEM } & \multicolumn{7}{|c|}{ Significance Level } \\
\hline & Sub & Mes & Sub & Mes & Sub & Mes & Sub & Mes & & B & $\mathrm{D}$ & FD & $\mathrm{B} \times \mathrm{D}$ & $\mathrm{B} \times \mathrm{FD}$ & $\mathrm{D} \times \mathrm{FD}$ & $\mathrm{B} \times \mathrm{D} \times \mathrm{FD}$ \\
\hline Total fatty acids & 496 & 603 & 473 & 558 & 455 & 442 & 436 & 532 & 32.5 & ** & ns & $* *$ & ns & ns & ns & ns \\
\hline \multicolumn{17}{|l|}{ Fatty acids } \\
\hline $12: 0$ & $0.068^{\mathrm{ac}}$ & $0.084^{\mathrm{bc}}$ & $0.064^{\mathrm{a}}$ & $0.076^{c}$ & $0.065^{\mathrm{ac}}$ & $0.103^{d}$ & $0.068^{\mathrm{ab}}$ & $0.076^{\mathrm{abc}}$ & 0.006 & ns & ns & $* * *$ & ns & * & $* *$ & $* *$ \\
\hline$i-14: 0$ & ND & $0.115^{\mathrm{a}}$ & ND & $0.075^{\mathrm{b}}$ & ND & $0.138^{c}$ & ND & $0.068^{c}$ & 0.007 & ns & $* * *$ & $* * *$ & ns & ns & $* * *$ & ns \\
\hline $14: 0$ & $3.52^{\mathrm{a}}$ & $3.23^{\mathrm{ab}}$ & $3.50^{\mathrm{a}}$ & $3.11^{b}$ & $3.00^{\mathrm{b}}$ & $3.52^{\mathrm{a}}$ & $3.20^{\mathrm{ab}}$ & $3.27^{\mathrm{ab}}$ & 0.175 & ns & ns & ns & ns & $* * *$ & ns & ns \\
\hline $14: 1 c 9$ & $0.726^{\mathrm{a}}$ & $0.138^{\mathrm{b}}$ & $0.803^{\mathrm{ac}}$ & $0.138^{\mathrm{b}}$ & $0.966^{c}$ & $0.166^{\mathrm{b}}$ & $1.16^{c}$ & $0.167^{b}$ & 0.072 & $* *$ & ns & $* * *$ & ns & $* *$ & ns & ns \\
\hline$i-15: 0$ & $0.280^{\mathrm{a}}$ & $0.331^{b}$ & $0.141^{\mathrm{c}}$ & $0.193^{\mathrm{d}}$ & $0.241^{\mathrm{e}}$ & $0.318^{\mathrm{ab}}$ & $0.157^{\mathrm{cd}}$ & $0.187^{\mathrm{d}}$ & 0.014 & ns & $* * *$ & $* * *$ & ns & ns & ns & * \\
\hline$a-15: 0$ & $0.252^{\mathrm{a}}$ & $0.461^{b}$ & $0.188^{\mathrm{ad}}$ & $0.326^{c}$ & $0.233^{\mathrm{ad}}$ & $0.420^{\mathrm{b}}$ & $0.176^{d}$ & $0.315^{\mathrm{e}}$ & 0.023 & ns & $* * *$ & $* * *$ & ns & ns & * & ns \\
\hline $15: 0$ & $0.429 \mathrm{ac}$ & $0.686^{b}$ & $0.496^{a}$ & $0.585^{c}$ & $0.456^{\mathrm{ad}}$ & $0.711^{\mathrm{b}}$ & $0.387^{d}$ & $0.515^{\mathrm{ac}}$ & 0.032 & ns & * & $* * *$ & * & ns & $* * *$ & ns \\
\hline$i-16: 0$ & $0.322^{\mathrm{a}}$ & $0.483^{b}$ & $0.182^{\text {de }}$ & $0.271^{\mathrm{ae}}$ & $0.311^{\mathrm{a}}$ & $0.405^{c}$ & $0.171^{\mathrm{d}}$ & $0.235^{\mathrm{e}}$ & 0.024 & ns & $* * *$ & $* * *$ & ns & $* *$ & $* * *$ & ns \\
\hline $16: 0$ & $27.3^{\mathrm{ad}}$ & $25.9^{\mathrm{cd}}$ & $26.5^{\text {acd }}$ & $25.6^{\mathrm{cd}}$ & $23.7^{b}$ & $26.0^{\text {acd }}$ & $25.0^{\mathrm{bc}}$ & $26.3^{d}$ & 0.559 & * & ns & ns & ns & $* * *$ & ns & ns \\
\hline $16: 1 c 7$ & $0.259^{a}$ & $0.398^{c}$ & $0.262^{a}$ & $0.350^{\mathrm{cd}}$ & $0.319 \mathrm{bd}$ & $0.373^{\mathrm{cd}}$ & $0.283^{a b c}$ & $0.343^{d}$ & 0.016 & ns & * & $* * *$ & ns & $* * *$ & ns & * \\
\hline $16: 1 c 9$ & $4.08^{a}$ & $0.993^{b}$ & $3.78^{a}$ & $1.01^{\mathrm{b}}$ & $4.94^{\mathrm{b}}$ & $1.04^{b}$ & $5.36^{c}$ & 1.14 & 0.227 & $* * *$ & ns & $* * *$ & ns & $* * *$ & ns & ns \\
\hline$i-17: 0$ & 0.285 & 0.353 & 0.240 & 0.321 & 0.314 & 0.382 & 0.252 & 0.319 & 0.014 & ns & $* * *$ & $* * *$ & ns & ns & ns & ns \\
\hline$a-17: 0$ & $1.31^{\mathrm{a}}$ & $1.16^{b}$ & $1.13^{b}$ & $1.01^{\mathrm{c}}$ & $1.46^{\mathrm{d}}$ & $0.973^{c}$ & $1.35^{\mathrm{a}}$ & $0.928^{c}$ & 0.034 & ns & $* * *$ & $* * *$ & ns & $* * *$ & ns & ns \\
\hline $17: 0$ & $0.920^{\mathrm{a}}$ & $1.59^{b}$ & $1.19 c$ & $1.65^{b}$ & $0.851^{\mathrm{a}}$ & $1.46^{\mathrm{ef}}$ & $0.729 d$ & $1.28^{\mathrm{cf}}$ & 0.041 & $* * *$ & ns & $* * *$ & $* * *$ & ns & ** & * \\
\hline $17: 1 c 9$ & $0.761^{\mathrm{a}}$ & $0.381^{b}$ & $1.01^{c}$ & $0.412^{\mathrm{b}}$ & $0.947^{c}$ & $0.386^{b}$ & $0.912^{c}$ & $0.383^{b}$ & 0.039 & ns & ns & $* * *$ & * & ns & * & $* *$ \\
\hline$i-18: 0$ & 0.243 & 0.249 & 0.181 & 0.187 & 0.210 & 0.222 & 0.148 & 0.191 & 0.012 & * & $* * *$ & $* *$ & ns & ns & ns & ns \\
\hline $18: 0$ & 14.5 & 32.2 & 12.9 & 30.8 & 11.4 & 29.3 & 9.75 & 27.8 & 0.859 & $* * *$ & * & $* * *$ & ns & ns & ns & ns \\
\hline $18: 1 t 6-t 8$ & 0.118 & 0.187 & 0.184 & 0.245 & 0.136 & 0.237 & 0.154 & 0.231 & 0.019 & ns & * & $* * *$ & ns & ns & ns & ns \\
\hline $18: 1 t 9$ & 0.232 & 0.252 & 0.246 & 0.284 & 0.240 & 0.272 & 0.269 & 0.289 & 0.012 & ns & * & $* * *$ & ns & ns & ns & ns \\
\hline $18: 1 t 10$ & $0.198^{a}$ & $0.274^{b}$ & $1.04^{c}$ & 0.979 c & $0.248^{\mathrm{ab}}$ & $0.302^{\mathrm{ab}}$ & $0.403^{\mathrm{ab}}$ & $0.432^{\mathrm{ab}}$ & 0.089 & $* *$ & $* * *$ & ns & $* * *$ & ns & * & ns \\
\hline $18: 1 t 11$ & $1.41^{\mathrm{ab}}$ & $2.21^{c}$ & $1.36^{\mathrm{a}}$ & $2.20^{c}$ & $1.88^{\mathrm{bc}}$ & $2.90^{\mathrm{d}}$ & $1.74^{\mathrm{ab}}$ & $2.83^{\mathrm{d}}$ & 0.139 & $* * *$ & ns & $* * *$ & ns & * & ns & ns \\
\hline $18: 1 t 12$ & $0.123^{a}$ & $0.297 \mathrm{~cd}$ & $0.202^{b}$ & $0.270^{c}$ & $0.196^{b}$ & $0.338^{d}$ & $0.194^{b}$ & $0.339 d$ & 0.021 & $* *$ & ns & $* * *$ & ns & ns & ns & * \\
\hline $18: 1 c 9$ & 33.4 & 20.8 & 34.8 & 21.7 & 37.3 & 22.2 & 37.3 & 24.2 & 0.850 & $* * *$ & ns & $* * *$ & ns & ns & ns & ns \\
\hline 18:1c11 & 4.13 & 2.30 & 3.70 & 2.51 & 4.32 & 2.21 & 4.53 & 2.75 & 0.233 & ns & ns & $* * *$ & ns & ns & ns & ns \\
\hline $18: 1 c 12$ & 0.761 & 0.601 & 0.721 & 0.625 & 0.805 & 0.629 & 0.841 & 0.674 & 0.036 & * & ns & $* * *$ & ns & ns & ns & ns \\
\hline $18: 1 c 13$ & $0.277^{\mathrm{a}}$ & $0.111^{b}$ & $0.388^{c}$ & $0.130^{\mathrm{b}}$ & $0.405^{c}$ & $0.114^{\mathrm{b}}$ & $0.521^{\mathrm{d}}$ & $0.145^{\mathrm{b}}$ & 0.031 & $* *$ & $* *$ & $* * *$ & ns & ** & * & ns \\
\hline $18: 1 t 16+c 14$ & $0.250^{\mathrm{a}}$ & $0.353^{b}$ & $0.198^{c}$ & $0.282^{\mathrm{a}}$ & $0.260^{a}$ & $0.328^{b}$ & $0.228^{\mathrm{ac}}$ & $0.332^{b}$ & 0.014 & ns & $* *$ & $* * *$ & * & ns & ns & ns \\
\hline 18:1c15 & 0.124 & 0.127 & 0.141 & 0.143 & 0.131 & 0.106 & 0.121 & 0.129 & 0.009 & ns & ns & ns & ns & ns & ns & ns \\
\hline
\end{tabular}




\begin{tabular}{|c|c|c|c|c|c|c|c|c|c|c|c|c|c|c|c|c|}
\hline & \multicolumn{4}{|c|}{ Alentejana } & \multicolumn{4}{|c|}{ Barrosã } & \multirow{2}{*}{\multicolumn{8}{|c|}{ Significance Level }} \\
\hline & \multicolumn{2}{|c|}{ HS } & \multicolumn{2}{|c|}{ LS } & \multicolumn{2}{|c|}{ HS } & \multicolumn{2}{|c|}{ LS } & & & & & & & & \\
\hline & Sub & Mes & Sub & Mes & Sub & Mes & Sub & Mes & SEM & B & $\mathrm{D}$ & FD & $B \times D$ & $\mathrm{~B} \times \mathrm{FD}$ & $\mathrm{D} \times \mathrm{FD}$ & $\mathrm{B} \times \mathrm{D} \times \mathrm{FD}$ \\
\hline $18: 2 c 9, t 11^{\S}$ & $0.448^{\mathrm{a}}$ & $0.260^{\mathrm{b}}$ & $0.500^{\mathrm{a}}$ & $0.225^{\mathrm{b}}$ & $0.873^{c}$ & $0.439^{a}$ & $0.900^{c}$ & $0.428^{a}$ & 0.034 & $* * *$ & ns & $* * *$ & ns & $* * *$ & ns & ns \\
\hline $18: 2 t 11, c 15$ & ND & $0.118^{a}$ & ND & $0.066^{\mathrm{b}}$ & ND & $0.132^{\mathrm{a}}$ & ND & $0.072^{\mathrm{b}}$ & 0.014 & ns & $* *$ & $* * *$ & ns & ns & $* *$ & ns \\
\hline $18: 2 n-6$ & $1.49^{a}$ & $1.87 \mathrm{ce}$ & $2.29^{\mathrm{be}}$ & $2.70^{\text {de }}$ & $1.70^{\mathrm{ac}}$ & $1.94^{\mathrm{c}}$ & $1.82^{\mathrm{c}}$ & $2.10^{\mathrm{e}}$ & 0.104 & * & $* * *$ & $* * *$ & $* * *$ & * & ns & ns \\
\hline $18: 3 n-3$ & $0.273^{\mathrm{ac}}$ & $0.323^{\mathrm{b}}$ & $0.259^{\mathrm{ac}}$ & $0.274^{\mathrm{ac}}$ & $0.324^{\mathrm{b}}$ & $0.379^{d}$ & $0.248^{a}$ & $0.278^{c}$ & 0.012 & * & $* * *$ & $* * *$ & $* *$ & ns & * & ns \\
\hline 19:1 & 0.254 & 0.193 & 0.264 & 0.186 & 0.335 & 0.192 & 0.301 & 0.211 & 0.057 & ns & ns & $* * *$ & ns & ns & ns & ns \\
\hline $20: 0$ & $0.093^{a}$ & $0.178^{\mathrm{b}}$ & $0.081^{\mathrm{ac}}$ & $0.172^{\mathrm{b}}$ & $0.079^{a}$ & $0.192^{\mathrm{b}}$ & $0.060^{c}$ & $0.170^{\mathrm{b}}$ & 0.010 & ns & * & $* * *$ & ns & * & ns & ns \\
\hline 20:1c11 & 0.146 & 0.096 & 0.154 & 0.105 & 0.169 & 0.117 & 0.196 & 0.133 & 0.012 & $* *$ & ns & $* * *$ & ns & ns & ns & ns \\
\hline $20: 3 n-6$ & ND & 0.062 & ND & 0.058 & ND & 0.070 & ND & 0.066 & 0.004 & ns & ns & $* * *$ & ns & ns & ns & ns \\
\hline $20: 4 n-6$ & $0.033^{a}$ & $0.045^{\mathrm{abc}}$ & $0.034^{\mathrm{ac}}$ & $0.058^{b}$ & $0.049 c$ & $0.046^{\mathrm{abc}}$ & $0.045^{\mathrm{abc}}$ & $0.048^{\mathrm{abc}}$ & 0.006 & ns & ns & * & ns & * & ns & ns \\
\hline
\end{tabular}

\$This peak also includes minor amounts of the $t 7, c 9$ and $t 8, c 10$ CLA isomers.

$\mathrm{ND}=$ not detected; $\mathrm{B}=$ breed; $\mathrm{D}=$ diet; $\mathrm{FD}=$ fat depot. Significance level: not significant (ns), $P>0.05 ;{ }^{*}, P<0.05 ;{ }^{* *}, P<0.01 ;{ }^{* * *}, P<0.001 ;$ means in the same row with different superscripts are significantly different $(P<0.05)$.

Table 4. CLA isomeric profile ( $\mathrm{mg} / \mathrm{l} 00 \mathrm{~g}$ fatty acids) of subcutaneous (Sub) and mesenteric (Mes) fats from Alentejana and Barrosã bulls fed high (HS) or low silage (LS) diets.

\begin{tabular}{|c|c|c|c|c|c|c|c|c|c|c|c|c|c|c|c|c|}
\hline & \multicolumn{4}{|c|}{ Alentejana } & \multicolumn{4}{|c|}{ Barrosã } & \multirow{2}{*}{\multicolumn{8}{|c|}{ Significance Level }} \\
\hline & \multicolumn{2}{|c|}{ HS } & \multicolumn{2}{|c|}{ LS } & \multicolumn{2}{|c|}{ HS } & \multicolumn{2}{|c|}{ LS } & & & & & & & & \\
\hline & Sub & Mes & Sub & Mes & Sub & Mes & Sub & Mes & SEM & B & $\mathrm{D}$ & FD & $B \times D$ & $\mathrm{~B} \times \mathrm{FD}$ & $\mathrm{D} \times \mathrm{FD}$ & $\mathrm{B} \times \mathrm{D} \times \mathrm{FD}$ \\
\hline Total CLA & $0.516^{\mathrm{a}}$ & $0.337^{c}$ & $0.479^{a}$ & $0.285^{c}$ & $0.985^{\mathrm{b}}$ & $0.524^{a}$ & $0.992^{\mathrm{b}}$ & $0.501^{\mathrm{a}}$ & 0.036 & $* * *$ & ns & $* * *$ & ns & ** & ns & ns \\
\hline$t 12, t 14$ & $3.17^{\mathrm{ac}}$ & $5.77^{\mathrm{d}}$ & $2.85^{\mathrm{ac}}$ & $4.22^{\mathrm{bc}}$ & $4.41^{\mathrm{b}}$ & $7.18^{e}$ & $3.56^{c}$ & $6.06^{\mathrm{d}}$ & 0.400 & $* * *$ & $* *$ & $* * *$ & ns & ns & * & ns \\
\hline$t 11, t 13$ & $6.91^{\mathrm{ab}}$ & $15.8^{c}$ & $5.18^{\mathrm{a}}$ & $10.9^{\mathrm{d}}$ & $9.09^{b d}$ & $20.0^{\mathrm{e}}$ & $5.21^{\mathrm{a}}$ & $13.2^{\mathrm{f}}$ & 0.695 & $* * *$ & $* * *$ & $* * *$ & ns & $* *$ & $* * *$ & ns \\
\hline$t 10, t 12$ & $4.69^{\mathrm{acd}}$ & $5.44^{\mathrm{cd}}$ & $7.36^{\mathrm{b}}$ & $7.01^{\mathrm{b}}$ & $6.48^{\mathrm{bc}}$ & $4.57^{\mathrm{d}}$ & $6.34^{\mathrm{bc}}$ & $6.57^{b}$ & 0.525 & ns & $* *$ & ns & ns & ns & ns & $* *$ \\
\hline$t 9, t 11$ & $10.5^{\mathrm{a}}$ & $12.5^{\mathrm{a}}$ & $10.1^{\mathrm{a}}$ & $10.1^{\mathrm{a}}$ & $18.6^{\text {bd }}$ & $14.6^{\mathrm{cd}}$ & $14.3^{\mathrm{abd}}$ & $16.9^{\mathrm{d}}$ & 2.90 & $* *$ & ns & ns & ns & ns & ns & * \\
\hline$t 8, t 10$ & $3.22^{\mathrm{ac}}$ & $5.26^{\mathrm{bc}}$ & $3.14^{\mathrm{ac}}$ & $3.53^{c}$ & $6.05^{\mathrm{b}}$ & $5.25^{\mathrm{b}}$ & $5.31^{b}$ & $4.29 \mathrm{ac}$ & 0.589 & $* *$ & ns & ns & ns & $* *$ & ns & $\mathrm{ns}$ \\
\hline$t 7, t 9$ & $5.41^{\mathrm{a}}$ & $6.22^{\mathrm{ac}}$ & $4.42^{\mathrm{ac}}$ & $5.23^{\mathrm{ac}}$ & $9.59^{b}$ & $5.30^{c}$ & $4.97 \mathrm{ac}$ & $5.35^{\mathrm{a}}$ & 0.670 & ns & $* *$ & ns & ns & ** & $* *$ & ** \\
\hline$t 6, t 8$ & $2.50^{\mathrm{ac}}$ & $4.01^{\mathrm{d}}$ & $2.02^{\mathrm{a}}$ & $1.89^{a}$ & $4.09 \mathrm{bcd}$ & $3.97^{\mathrm{bd}}$ & $3.38^{\mathrm{cd}}$ & $1.85^{\mathrm{a}}$ & 0.483 & ns & $* * *$ & ns & ns & * & * & ns \\
\hline total trans,trans & $36.4^{\mathrm{a}}$ & $55.0^{\mathrm{bc}}$ & $35.1^{\mathrm{a}}$ & $42.9 \mathrm{ac}$ & $64.7 \mathrm{bc}$ & $60.9 c$ & $43.1^{\mathrm{a}}$ & $54.2^{\mathrm{b}}$ & 4.25 & $* * *$ & $* *$ & $* *$ & ns & ns & ns & * \\
\hline$c / t 12,14$ & $2.86^{\mathrm{a}}$ & $2.26^{\mathrm{a}}$ & $3.31^{\mathrm{ab}}$ & $2.11^{\mathrm{a}}$ & $4.48^{\mathrm{b}}$ & $2.41^{\mathrm{a}}$ & $4.40^{\mathrm{b}}$ & $2.19^{a}$ & 0.500 & * & ns & $* * *$ & ns & ns & ns & ns \\
\hline$t 11, c 13$ & $12.9^{a}$ & $14.6^{\mathrm{d}}$ & $5.78^{\mathrm{b}}$ & $4.96^{\mathrm{b}}$ & $17.9^{c}$ & $17.7^{\mathrm{c}}$ & $11.9^{\text {ad }}$ & $10.1^{\mathrm{a}}$ & 1.05 & $* * *$ & $* * *$ & ns & ns & ns & $* *$ & ns \\
\hline$c 11, t 13$ & $3.33^{a}$ & $0.749^{\mathrm{d}}$ & $4.19^{a b}$ & $0.429^{d}$ & $5.35^{\mathrm{b}}$ & $0.734^{\mathrm{d}}$ & $6.97^{c}$ & $0.893^{d}$ & 0.495 & $* * *$ & ns & $* * *$ & ns & $* *$ & * & ns \\
\hline$t 10, c 12$ & $7.36^{\mathrm{a}}$ & $4.05^{\mathrm{d}}$ & $14.9^{\mathrm{b}}$ & $10.3^{\mathrm{ac}}$ & $11.1^{\mathrm{c}}$ & $3.62^{\mathrm{d}}$ & $14.5^{\mathrm{b}}$ & $5.27^{\mathrm{ad}}$ & 1.07 & ns & $* * *$ & $* * *$ & * & $* * *$ & ns & ns \\
\hline$c 9, t 11$ & $399 a$ & $230^{c}$ & $338^{a}$ & $187^{c}$ & $795^{b}$ & $396^{\text {bcd }}$ & $806^{\mathrm{b}}$ & $377^{\mathrm{ad}}$ & 30.8 & $* * *$ & ns & $* * *$ & ns & $* * *$ & ns & ns \\
\hline$t 8, c 10$ & $8.59^{a}$ & $9.44^{\mathrm{ac}}$ & $8.28^{\mathrm{a}}$ & $6.54^{\mathrm{a}}$ & $15.4^{\mathrm{b}}$ & $13.5^{\mathrm{bc}}$ & $16.0^{\mathrm{b}}$ & $11.9^{c}$ & 0.993 & $* * *$ & ns & $* *$ & ns & * & * & ns \\
\hline$t 7, c 9$ & 40.9ae & $21.3^{\mathrm{d}}$ & $63.6^{\mathrm{b}}$ & $30.9^{\mathrm{de}}$ & $62.7^{b}$ & $29.5^{\mathrm{d}}$ & $77.5^{c}$ & $39.1^{\mathrm{e}}$ & 3.42 & $* * *$ & $* * *$ & $* * *$ & ns & * & * & ns \\
\hline total cis/trans & $475^{\mathrm{a}}$ & $282^{c}$ & $438^{a}$ & $243^{c}$ & $912^{\mathrm{b}}$ & $463^{a}$ & $938^{\mathrm{b}}$ & $447^{a}$ & 34.6 & $* * *$ & ns & $* * *$ & ns & $* * *$ & ns & ns \\
\hline$c 9, c 11$ & $5.46^{\mathrm{ad}}$ & $2.14^{\mathrm{cd}}$ & $6.50^{\mathrm{a}}$ & $1.95^{\mathrm{cd}}$ & $10.1^{\mathrm{b}}$ & $2.86^{\mathrm{cd}}$ & $11.1^{\mathrm{b}}$ & $3.40^{\mathrm{d}}$ & 0.777 & $* * *$ & ns & $* * *$ & ns & $* *$ & ns & ns \\
\hline
\end{tabular}

$\mathrm{D}=$ diet; $\mathrm{B}=$ breed; $\mathrm{FD}=$ fat depot. Significance level: not significant $(\mathrm{ns}), \mathrm{P}>0.05 ;{ }^{*}, \mathrm{P}<0.05 ;{ }^{* *}, \mathrm{P}<0.01 ; * * *, \mathrm{P}<0.001$; means in the same row with different superscripts are significantly different $(\mathrm{P}<0.05)$.

There were significant effects of breed $(P<0.001)$ and fat depot $(P<0.001)$, as well as its respective interaction $(P<0.01)$, on the total SFA and MUFA proportions (Table 5). Total TFA were not affected by diet $(P>0.05)$. In contrast, this lipid class was influenced by the fat depot $(P<0.001)$ and its interaction with breed $(P<0.05)$, with higher values in mesenteric fat of Bar- rosã bulls and subcutaneous fat of Alentejana bulls. Fat depot, diet, and the interactions breed $\times$ diet and breed $\times$ fat depot, were major determinants on PUFA (at least, $P<0.05$ ), with higher percentages observed for low silage fed animals. The high silage diet promoted higher percentages of BCFA in both fat depots $(P<0.001)$. An interaction between breed and fat depot 
was also observed $(P<0.001)$ due to higher proportions of these fatty acids in the mesenteric fat of Alentejana bulls. Concerning the $\Delta 9$-indices (stearoyl-CoA desaturase activity indicators), an interaction between breed and diet $(P<0.001)$ was observed, with subcutaneous fat of Barrosã bulls, showing the highest values. The only exception was the $\Delta 9-18$ index, which was influenced by breed $(P<0.001)$, fat depot $(P<0.001)$ and diet $(P<0.05)$. This former index was higher in subcutaneous fat of low silage fed Barrosã bulls. In contrast to the desaturation indices, neither breed nor diet influenced the elongation ratio, which showed to be strongly influenced by the fat $\operatorname{depot}(P<0.001)$, as well as its interaction with breed $(P<0.05)$.

\section{MUFA discriminate the subcutaneous fat from the mesenteric fat}

A PCA was applied to a data set of cellularity and fatty acid composition parameters in order to describe the variability of the pooled data into two dimensions (Fig. 2A). The score plot of the first two components explains $58.1 \%$ of the total variability, with $45.3 \%$ for PC1 and $12.8 \%$ for PC2 (Table 6). The score plot showed a clear separation of adipocytes number from MUFA, which in turn were closely associated with adipocytes area. The cell number was located near the arachidonic acid and some of the 18:2n-6 ruminal biohydrogenation related fatty acids (18:1t11 and 18:t12). Most of the MUFA were allocated on the left side of the plot (quadrants $a$ and $d$ ), clearly separated from the other variables, showing negative scores for the PC1 and little influence on the PC2.

Table 5. Partial sums of fatty acids ( $\mathrm{g} / \mathrm{l} 00 \mathrm{~g}$ fatty acids) and $\Delta 9$-indices of subcutaneous (Sub) and mesenteric (Mes) fats from Alentejana and Barrosã bulls fed high (HS) or low silage (LS) diets.

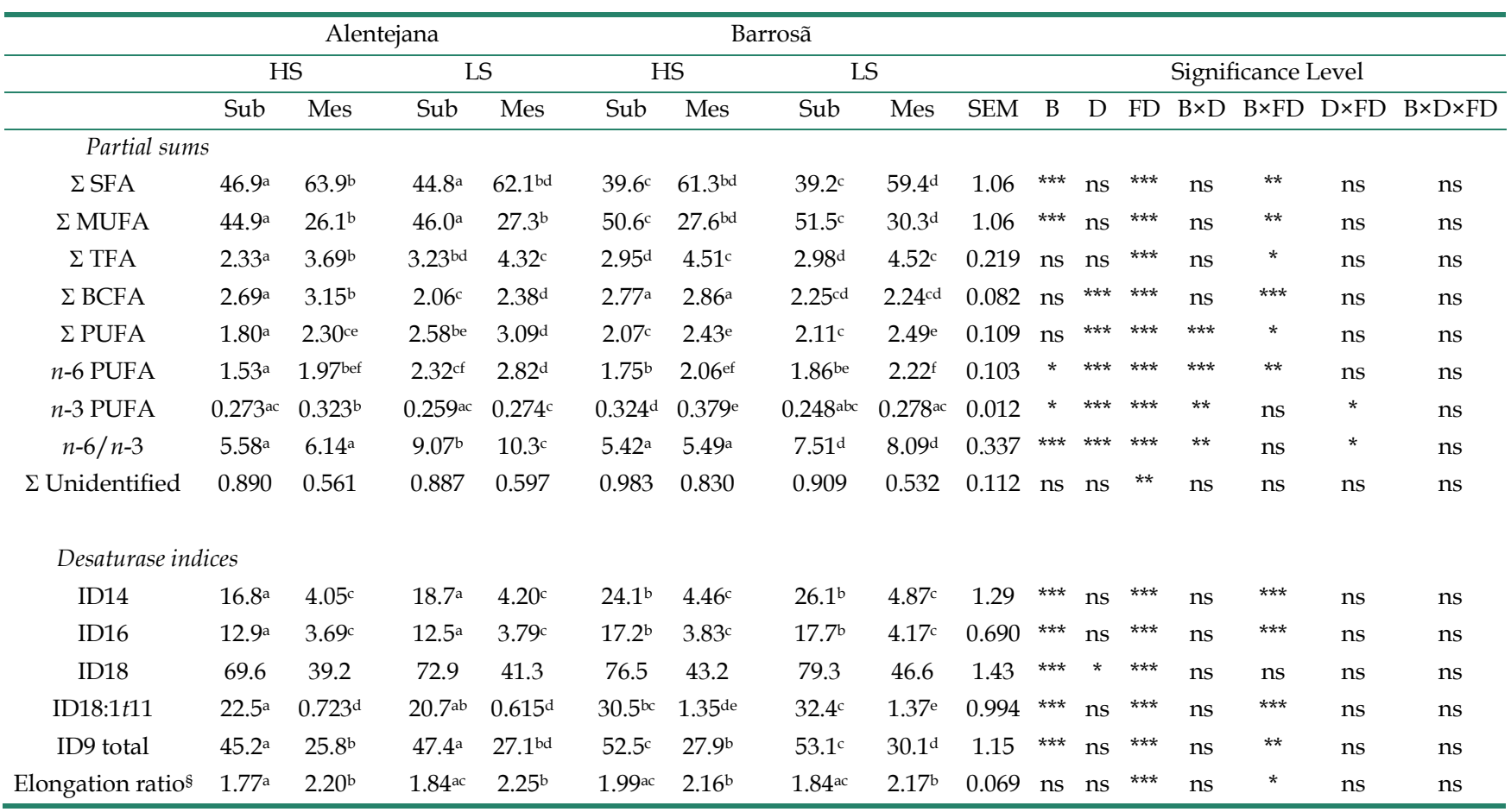

$\Sigma \mathrm{SFA}=$ sum of 14:0, 15:0, 16:0, 17:0, 18:0 and 20:0; $\Sigma$ MUFA = sum of 14:1c9, 16:1c7, 16:1c9, 17:1c9, 18:1c9, 18:1c11, 18:1c12, 18:1c13, 18:1c15 19:1 and 20:1c11; $\Sigma$ TFA = sum of 18:1t6-t8, 18:1t9, 18:1t10, 18:1t11, 18:1t12, 18:1t16+c14 and 18:2t11c15; $\Sigma$ PUFA = sum of 18:2n-6, 18:3n-3, 20:3n-6 and 20:4n-6; $\Sigma$ BCFA = sum of $i-14: 0, i-15: 0, a-15: 0, i-16: 0, i-17: 0, a-17: 0$ and $i-18: 0 ; \Sigma n-6=$ sum of $18: 2 n-6,20: 2 n-6$ and $20: 4 n-6 ; \Sigma n-3=$

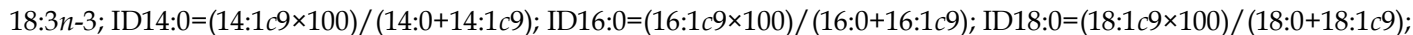

ID18:1t11 $=(18: 2 c 9, t 11 \times 100) /(18: 1 t 11+18: 2 c 9, t 11)$. ID9 total $=(14: 1 c 9+16: 1 c 9+18: 1 c 9+$ CLA $c 9, t 11) \times 100 /(14: 1 c 9+16: 1 c 9+18: 1 c 9+$ CLA $c 9, t 11+14: 0+16: 0+18: 0+18: 1 t 11)$

$\S$ Elongation ratio $=$

$(18: 0+18: 1 t 6-t 8+18: 1 t 9+18: 1 t 10+18: 1 t 11+18: 1 t 12+18: 1 c 9+18: 1 c 11+18: 1 c 12+18: 1 c 13+18: 1 t 16+c 14+18: 1 c 15) /(16: 0+16: 1 c 7+16: 1 c 9)$

$\mathrm{D}=$ diet; $\mathrm{B}=$ breed; $\mathrm{FD}=$ fat depot. Significance level: not significant $(\mathrm{ns}), P>0.05 ;{ }^{*}, P<0.05 ;{ }^{* *}, P<0.01 ;{ }^{* * *}, P<0.001$; means in the same row with different superscripts are significantly different $(P<0.05)$. 

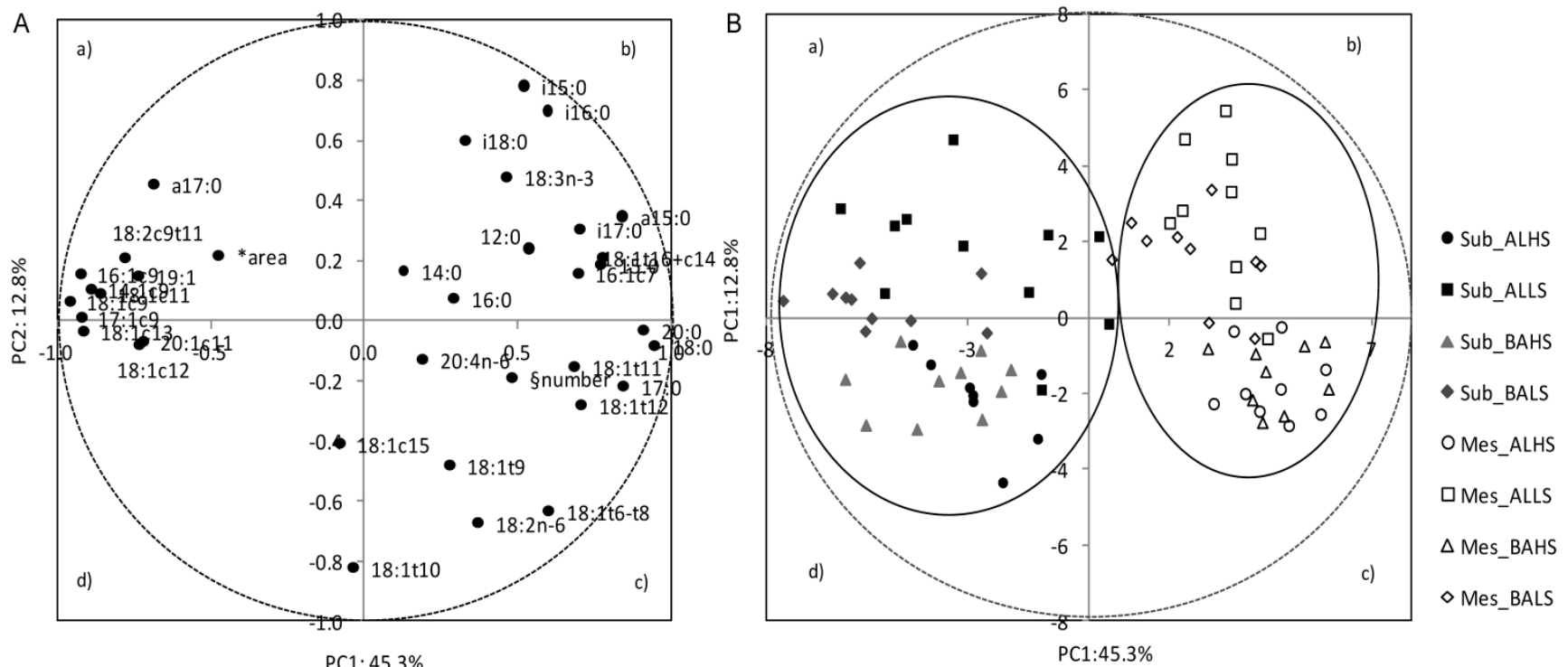

Figure 2. Loading plot of the first and second principal components (PC) of the pooled data $(A)$ and component's score vectors (B) for subcutaneous (Sub) and mesenteric (Mes) fats from Alentejana (AL) and Barrosã (BA) bulls fed high (HS) or low (LS) silage diets. *Adipocytes area; $\S$ Adipocytes number

The PC2 clearly distinguished BCFA, located in the upper part of the plot, from adipocytes number, located in the lower part of the graphic (quadrant $c$ ). In quadrant $b$, a cluster was defined by $a-15: 0, i-16: 0$, $16: 1 c 7,18: 1 t 16+c 14$ and $i-17: 0$ fatty acids.

The score plot depicted in Figure 2B shows the location of subcutaneous and mesenteric adipose fats in the multivariate space of the first two PCs. These scores were notably arranged in two clusters, corresponding to both fat depots. In contrast, no clear discrimination between breeds, Alentejana and Barrosã, or diets, $30 / 70 \%$ and $70 / 30 \%$ of silage and concentrate, was achieved.

\section{Discussion}

The economical and physiological importance of fat deposition in meat animal production has long been recognized [28; 4]. Subcutaneous fat, along with the intermuscular fat, is the largest adipose tissue depot [29] with the highest lipogenic activity [30], whereas mesenteric fat displays distinctive immune-response potential [31; 32]. Nonetheless, information concerning the biology and regulation of each fat depot is limited.

In the present study, live slaughter weight was determined by breed, as Alentejana and Barrosã bulls are quite distinct in morphological characteristics [11]. As for the diet, it showed no impact on live slaughter weight. Regarding the deposition of adipose tissue, there was no influence from breed or diet on the subcutaneous fat. The analysis of the visceral fat par- tioning revealed diet as the major influencing factor, in particular for mesenteric fat in low silage fed animals. The critical factor affecting glucose levels in plasma was the breed, with higher values in Alentejana than in Barrosã bulls. It is well known that ruminants have typical insulin resistance compared to monogastric animals. Insulin concentrations were affected by diet, as low forage diets fed to both breeds promoted higher values of this hormone. In ruminants, dietary carbohydrates are fermented into volatile fatty acids by ruminal microorganisms, and the propionate formed is used as a primary precursor for gluconeogenesis [33]. Therefore, propionate from rumen fermentation is largely associated with body fat deposition, as it promotes lipogenesis through the secretion of insulin.

Leptin is synthesized and released into the bloodstream in direct proportion to the amount of body fat, reflecting primarily the triacylglycerols content of lipid depots, but also functioning as a sensor of energy balance [34; 35]. The systemic leptin levels are strongly associated with mRNA levels in subcutaneous adipose tissue and cellularity [36; 37]. Herein, plasma leptin levels were unchanged across dietary groups, which is consistent with the lack of variation in subcutaneous fat tissue parameters.

IL-6, a primary pro-inflammatory interleukin, has been reported to be increased in fat animals [38]. In this study, plasma concentration of IL-6 was influenced by breed, with higher levels in Barrosã than in Alentejana bulls. Even if this might reflect the breed 
effect observed in one of the largest adipocyte classes (from 14400 to $16200 \mu \mathrm{m}^{2}$ ), it should be underlined that IL-6 is not exclusively adipose tissue-derived. In fact, only $10 \%$ of this cytokine is produced by adipocytes [39].

Table 6. Loadings for the first four principal components (PC).

\begin{tabular}{|c|c|c|c|c|}
\hline Variables & PC1 & PC2 & PC3 & PC4 \\
\hline $12: 0$ & 0.536 & 0.240 & -0.171 & 0.557 \\
\hline 14:0 & 0.128 & 0.167 & -0.662 & 0.561 \\
\hline$i-15: 0$ & 0.520 & 0.781 & 0.041 & 0.072 \\
\hline$a-15: 0$ & 0.839 & 0.349 & 0.025 & 0.057 \\
\hline $14: 1 c 9$ & -0.888 & 0.106 & -0.076 & 0.293 \\
\hline $15: 0$ & 0.769 & 0.188 & -0.162 & 0.432 \\
\hline$i-16: 0$ & 0.597 & 0.698 & 0.030 & 0.012 \\
\hline $16: 0$ & 0.291 & 0.076 & -0.737 & 0.182 \\
\hline$i-17: 0$ & 0.701 & 0.306 & 0.430 & 0.101 \\
\hline $16: 1 c 7$ & 0.697 & 0.159 & 0.263 & 0.474 \\
\hline $16: 1 c 9$ & -0.922 & 0.157 & -0.052 & 0.223 \\
\hline$a-17: 0$ & -0.685 & 0.456 & 0.137 & 0.202 \\
\hline $17: 0$ & 0.842 & -0.216 & -0.128 & -0.082 \\
\hline i18:0 & 0.329 & 0.601 & -0.024 & -0.337 \\
\hline $17: 1 c 9$ & -0.920 & 0.011 & -0.102 & 0.187 \\
\hline $18: 0$ & 0.944 & -0.082 & 0.068 & -0.218 \\
\hline $18: 1+6-t 8$ & 0.599 & -0.632 & 0.212 & 0.197 \\
\hline $18: 1+9$ & 0.278 & -0.479 & 0.585 & 0.177 \\
\hline $18: 1 t 10$ & -0.036 & -0.820 & -0.146 & 0.221 \\
\hline 18:1t11 & 0.683 & -0.151 & 0.426 & 0.162 \\
\hline $18: 1 t 12$ & 0.705 & -0.279 & 0.253 & 0.111 \\
\hline $18: 1 c 9$ & -0.957 & 0.065 & 0.083 & -0.002 \\
\hline 18:1c11 & -0.858 & 0.091 & 0.078 & 0.127 \\
\hline 18:1c12 & -0.732 & -0.078 & 0.328 & 0.162 \\
\hline 18:1c13 & -0.913 & -0.033 & 0.080 & 0.126 \\
\hline $18: 1 t 16+c 14$ & 0.775 & 0.212 & 0.365 & 0.148 \\
\hline 18:1c15 & -0.079 & -0.407 & -0.050 & 0.496 \\
\hline $18: 2 n-6$ & 0.370 & -0.671 & 0.075 & 0.222 \\
\hline 19:1 & -0.735 & 0.150 & 0.283 & 0.327 \\
\hline 20:0 & 0.908 & -0.030 & 0.203 & -0.020 \\
\hline $18: 3 n-3$ & 0.463 & 0.479 & 0.252 & 0.317 \\
\hline 20:1c11 & -0.719 & -0.067 & 0.290 & -0.085 \\
\hline $18: 2 c 9 t 11$ & -0.779 & 0.210 & 0.298 & 0.261 \\
\hline $20: 4 n-6$ & 0.189 & -0.127 & 0.169 & -0.094 \\
\hline Adipocytes area & -0.475 & 0.218 & 0.423 & -0.031 \\
\hline Adipocytes number & 0.480 & -0.188 & -0.412 & 0.009 \\
\hline Proportion of variance (\%) & 45.3 & 12.8 & 8.36 & 6.37 \\
\hline Cumulative variance (\%) & 45.3 & 58.1 & 66.5 & 72.8 \\
\hline
\end{tabular}

The histological characterization of fat depots showed no significant differences between breeds. Yet, fat depot was of extreme importance regarding cellularity and fatty acid profile. The increase of adi- pocytes area in the subcutaneous fat relatively to the mesenteric fat might be an indicator of an early adipocytes differentiation in this fat depot. Apart from this, no other significant effects were observed on fat depot cellularity. Concomitantly, there were also no significant differences across the experimental groups regarding plasma triacylglycerols.

The amount of adipose tissue in animals during growth is related both with hyperplasia and hypertrophy, although growth of different adipose tissues in cattle after birth is more attributable to adipocytes hypertrophy [40]. According to Robelin [28], subcutaneous adipose tissues have the highest relative growth and appear to be the youngest on a cellularity basis: small-sized cells, high and late hyperplasia, in comparison to internal fat depots. In the same experiment, kidney and peritoneal adipose tissues were used as representative of the internal fat depots [28]. Similar results have been reported by Mendizabal et al. [41] in a study concerning adipocytes size of subcutaneous and two visceral fat depots (omental and perirenal) from several bovine breeds. These authors observed that subcutaneous fat had smaller adipocytes than both omental and perirenal fat depots. Nevertheless, both studies failed to characterize the mesenteric fat depot. In contrast, our results showed that the subcutaneous fat had larger adipocytes than the chosen visceral fat depot, in accordance to Pike and Roberts [42] findings. Thus, the differential cellularity of mesenteric fat from other visceral fat depots could result from distinct lipogenic activities.

The relationship between adipocytes size and fat depot mass has been used to establish which process, hypertrophy, hyperplasia or both, is responsible for fat depot development [41]. A high correlation coefficient indicates the prevalence of hypertrophy, whereas a low correlation coefficient reveals the occurrence of both processes. The lack of a significant correlation points to hyperplasia as the main contributor to tissue development. In this study, the correlation coefficients between adipocytes area and fat depot mass might suggest that while the development of mesenteric fat may be due to both hypertrophy and hyperplasia, the subcutaneous fat development may be mostly attributed to hyperplasia.

The highest amount of fatty acids deposition was observed in the mesenteric fat of Alentejana breed. This might be due to differences in fatty acid deposition mechanisms between breeds, with Alentejana accumulating higher amounts of fatty acids within this internal fat depot. When comparing both adipose tissues, the mesenteric fat depot was more saturated and richer in TFA, PUFA and BCFA, whereas subcutaneous fat depot contained more MUFA. Diet was 
the major factor affecting PUFA and BCFA contents, while SFA and MUFA were breed-related. The high MUFA content in the subcutaneous adipose tissue was already reported [1], as a consequence of elevated stearoyl-CoA $\Delta 9$-desaturase activity. The ruminal transformation of dietary lipids plays a key role in determining the fatty acid composition of ruminant products. Diets containing high proportions of non-structural carbohydrates, as starch, but low amount of fiber promote usually less extensive biohydrogenation [43]. In fact, BCFA percentages in both fat depots were higher in high silage than in low silage fed animals. According to Aldai et al. [1], BCFA are higher in leaner animals and, in fact, no effect of breed was observed. This is concomitant with other parameters measured in this study, namely fat depots mass, cellularity or leptin levels. On the other hand, dietary starch is negatively correlated with $i-14: 0$, $i-15: 0$ and $i-16: 0$ fatty acids [44]. Indeed, the higher proportions of starch observed in low silage diet promoted a decrease in the aforementioned BCFA relatively to high silage diet.

Typically, feeding maize silages results in a high $n-6 / n-3$ ratio in body fat due to its high content in 18:2n-6 [45]. It also increases de novo synthesis of SFA from starch. In ruminants, the ratio of 18:0/18:2n-6 in the adipose tissue declines, as fattening proceeds [9]. Indeed, low silage fed Barrosã bulls showed the lowest value of the above mentioned ratio (5.41) in the subcutaneous fat, which reinforces the results regarding total fatty acids. Breed was also an important factor influencing CLA deposition and, in both fat depots, those changes occurred in parallel with vaccenic acid (18:1t11) variations. Shen et al. [46] and Dance et al. [47] reported breed-specific responses regarding vaccenic acid and CLA contents. Hishikawa et al. [7] and Gotoh et al. [48] suggested a distinct regulation of adipose tissue development promoted by differential expression of fat-related genes in subcutaneous and visceral adipose tissues. The $c 9, t 11$ CLA isomer content in adipose tissue derives from local biosynthesis from vaccenic acid by stearoyl-CoA $\Delta-9$ desaturase enzyme. Significant differences between breeds and fat depots were observed in the vaccenic acid, which were consequently reflected on the $c 9, t 11$ CLA isomer proportions. In a study by Garcia et al. [49], the proportion of CLA in the adipose tissue increased with fatness, as did vaccenic acid.

Lipids of forages and feedstuffs, mainly rich in $18: 3 n-3$ and $18: 2 n-6$, once in the rumen, are readily hydrolyzed to non-esterified fatty acids by microbial lipases. After hydrolysis, the released non-esterified PUFA undergo a series of enzymatic steps leading to the formation of more SFA, until 18:0 fatty acid. Dur- ing biohydrogenation, multiple intermediates of fatty acids are formed, such as CLA isomers and 18:1 trans- 11 . The $c 9, t 11$ CLA isomer was the most abundant across breeds, diets and fat depots, followed by the $t 7, c 9$, as reported by Fritsche et al. [50] for beef and milk. Breed showed a clear influence on CLA isomers' proportions, affecting eleven of the fifteen isomers. Dannenberger et al. [10] also reported significant differences between breeds on CLA isomers distribution in distinct fat depots. The influence of diet was also noticeable on the main CLA isomers. The low silage diet promoted lower proportions of $t 11, t 13$ and $t 11, c 13$ CLA isomers compared to the high silage diet. Similar results were described when pasture and concentrate diets were compared [10]. High silage feeding decreased the proportions of $t 10, c 12$ CLA isomer in comparison to low silage, which is in accordance with Dannenberger et al. [10]. Overall, the subcutaneous fat showed higher deposition of CLA isomers. This is consistent with reports on the highest lipogenic activity in this adipose tissue depot [30]. Nevertheless, Eguinoa and colleagues [51], in a study concerning the subcutaneous, intermuscular, omental and perirenal fat depots, reported that the visceral depots exhibited higher lipogenic enzyme activities per cell than the subcutaneous fat. However, when the catalytic activity per cell was adjusted for cell size, the subcutaneous depot had greater enzyme activities than omental and perirenal fats.

The higher and lower proportions of SFA and MUFA, respectively, in mesenteric fat, relatively to subcutaneous fat, suggest a higher lipogenic activity in the former. This explanation is in agreement with higher $\Delta 9$-desaturase indices, key indicators of lipogenic activity [52], found in the subcutaneous fat, in comparison to the mesenteric fat. The breed-related variations in the $\Delta 9$-desaturase indices observed in this study indicate a differential $\Delta 9$-desaturase activity, favoring Barrosã breed. This might be a consequence of a higher lipogenic activity in Barrosã, an early maturing breed, in comparison to Alentejana, known to be late maturing. In addition, fat depot and its interaction with breed, showed an influence on the elongation index. This suggests differential $\Delta 5$ and $\Delta 6$ desaturase activities between the subcutaneous and mesenteric fat depots. These enzymes are involved in the conversion of C18 PUFA to their long-chain derivatives.

The PCA established the relationships between cellularity and fatty acid composition of both adipose tissues, along with associations among fatty acids. This statistical approach confirmed the subcutaneous and mesenteric adipose tissues contrasting features, which arose from the analysis of variance. MUFA, 
closely associated with adipocytes area, was the major contributor for the distinction between fat depots which reinforces the concept of a differential metabolic and desaturase activity. Considering that $\Delta 9$ is the key enzyme converting SFA into MUFA, and bearing in mind the close association between most MUFA and adipocytes area, it would be plausible to speculate that higher adipocytes area found in the subcutaneous fat is responsible for a higher desaturation activity.

The genetic background strongly influences lipid incorporation, as shown through the fatty acid composition and CLA isomeric profile. However, contrarily to expected, the influence of breed on subcutaneous and mesenteric fat deposition and partitioning was not significant. Feeding different silage/concentrate ratios impacted on PUFA, BCFA and CLA isomers, which were the classes of fatty acids most sensitive to diet composition. Taking into account all results herein presented, the major factor determining lipid deposition is fat depot. Moreover, the distinct cellularity observed in subcutaneous and mesenteric fats from bulls might reflect a differential dynamics between hypertrophy and hyperplasia processes in these two adipose tissue depots.

\section{Acknowledgments}

The authors are grateful to Sandra Carvalho for assistance in microscope slides preparation and Dr. Shabir Najmudin for the careful revision of the manuscript. We also thank Clínica Médica e Diagnóstico Dr. Joaquim Chaves (Algés, Portugal) for the technical assistance concerning plasma metabolites determination. Financial support from Fundação para a Ciência e a Tecnologia (FCT) grant (PTDC/CVT/2006/66114) and individual fellowships to A.S.H. Costa (SFRH/BD/2009/61068), S.V. Martins (SFRH/BPD/2009/63019) and S.P. Alves (SFRH/BD/2007/37793) are acknowledged. Paula A. Lopes is a researcher from the FCT program "Ciência $2008^{\prime \prime}$.

\section{Abbreviation}

BCFA: branched chain fatty acids; CLA: conjugated linoleic acid; FAME: fatty acid methyl esters; IL-6: interleukin-6; KKCF: kidney knob and channel fat; MUFA: monounsaturated fatty acids; PCA: principal components analysis; PDO: protected designation of origin; PUFA: polyunsaturated fatty acids; SFA: saturated fatty acids; TFA: trans fatty acids.

\section{Conflict of Interests}

The authors have declared that no conflict of interest exists.

\section{References}

1. Aldai N, Nájera AI, Dugan MER et al. Characterization of intramuscular, intermuscular and subcutaneous adipose tissues in yearling bulls of different genetic groups. Meat Sci. 2007; 76: 682-691.

2. De Smet S, Raes K, Demeyer D. Meat fatty acid composition as affected by fatness and genetic factors: a review. Anim Res 2004; 53: 81-98.

3. Trayhurn P and Wood IS. Signaling role of adipose tissue: adipokines and inflammation in obesity. Biochem Soc T 2005; 33: 1078-1081.

4. Hood RL. Relationships among growth, adipose cell size, and lipid metabolism in ruminant adipose tissue. Fed Proc 1982; 41: 2555-2561.

5. Fain JN. Release of interleukins and other inflammatory cytokines by human adipose tissue is enhanced in obesity and primarily due to the nonfat cells. Vitam Horm 2006; 74: 443-477.

6. Vendrell J, Broch $\mathrm{M}$, Vilarrasa $\mathrm{N}$ et al. Resistin, adiponectin, ghrelin, leptin and proinflammatory cytokines: relationships in obesity. Obes Res 2004; 12: 962-971.

7. Hishikawa D, Hong YH, Roh SG et al. Identification of genes expressed differentially in subcutaneous and visceral fat of cattle, pig, and mouse. Physiol Genomics 2005; 21: 343-350.

8. Geay Y, Bauchart D, Hocquette JF et al. Effect of nutritional factors on biochemical, structural and metabolic characteristics of muscles in ruminants, consequences on dietetic value and sensorial qualities of meat. Reprod Nutr Devel 2001; 41:1-26.

9. Wood JD, Enser M, Fischer AV et al. Fat deposition, fatty acid composition and meat quality: a review. Meat Sci 2008; 78: 343-358.

10. Dannenberger D, Nuernberg $K$, Nuernberg $G$ et al. Effect of pasture vs. concentrate diet on CLA isomer distribution in different tissue lipids of beef cattle. Lipids 2005; 40: 589-598.

11. Fraústo da Silva M, Cardoso Lemos JP, Monteiro LS et al. Studies on growth and form: multivariate analysis of distribution of muscle and fat in Portuguese cattle breeds. Livest Prod Sci 1998; 55: 261-271.

12. Beja-Pereira A, Alexandrino P, Bessa I et al. Genetic characterization of Southwestern European bovine breeds: a historical and biogeographical reassessment with a set of 16 microsatellites. J Hered 2003; 94: 243-250.

13. Reis C, Navas D, Pereira $M$ et al. Growth hormone Alul polymorphism analysis in eight Portuguese bovine breeds. Arch Zootec 2001; 50: 41-48.

14. Alfaia CMM, Ribeiro VSS, Lourenço MRA et al. Fatty acid composition, conjugated linoleic acid isomers and cholesterol in beef from crossbred bullocks intensively produced and from Alentejana purebred bullocks reared according to Carnalentejana-PDO specifications. Meat Sci 2006; 72: 425-436.

15. GPP. Inquérito aos agrupamentos gestores de produtos com nomes protegidos DOP/IGP/ETG: 2006 e 2007. Lisboa: Gabinete de Planeamento e Políticas, Ministério da Agricultura, do Desenvolvimento Rural e das Pescas; 2009.

16. Alfaia CMM, Castro MLF, Martins SIV et al. Effect of slaughter season on fatty acid composition, conjugated linoleic acid isomers and nutritional value of intramuscular fat in Barrosã-PDO veal. Meat Sci 2007; 75: 44-52.

17. Alfaia CPM, Alves SP, Martins SIV et al. Effect of the feeding system on intramuscular fatty acids and conjugated linoleic acid isomers of beef cattle, with emphasis on their nutritional value and discriminatory ability. Food Chem 2009; 114: 939-946.

18. Martins AP, Lopes PA, Costa ASH et al. Differential mesenteric fat deposition in bovines fed on silage or concentrate is independent of glycerol membrane permeability. Animal 2011; 5: 1949-1956. 
19. Simões JA and Mendes I. Distribution of tissues in carcasses at the same proportion of total fat in Portuguese cattle breeds. Anim Res 2003; 52: 287-298.

20. Corino C, Di Giancamillo A, Rossi R et al. Dietary conjugated linoleic acid affects morphofunctional and chemical aspects of subcutaneous adipose tissue in heavy pigs. J Nutr 2005; 135 : 1444-1450.

21. Folch J, Lees M and Stanley GHS. A simple method for the isolation and purification of total lipids from animal tissues. J Biol Chem 1957; 226: 497-509.

22. Carlson LA. Extraction of lipids from human whole serum and lipoproteins from rat liver tissue with methylene chloride-methanol: a comparison with extraction with chloroform-methanol. Clin Chim Acta 1985; 149: 89-93.

23. Raes K, Smet $S$ and Demeyer D. Effect of double-muscling in Belgium Blue young bulls on the intramuscular fatty acid composition with emphasis on conjugated linoleic acid and polyunsaturated fatty acids. Anim Sci 2001; 73: 253-260.

24. Bessa RJB, Alves SP, Jerónimo E, Alfaia CM, Prates JAM, Santos-Silva J. Effect of lipid supplements on ruminal biohydrogenation intermediates and muscle fatty acids in lambs. Eur J Lipid Sci Technol 2007; 109: 868-878.

25. Rego O, Rosa H, Regalo $S$ et al. Seasonal changes of CLA isomers and other fatty acids of milk fat from grazing dairy herds in the Azores. J Agr Food Chem 2008; 88: 1855-1859.

26. Statistical Analysis Institute (SAS). SAS/STAT user's guide; Version 9.1. NC, USA: Cary. 2004.

27. Sturges HA. The choice of a class-interval. J Am Stat Assoc 1926; 21: 65-66.

28. Robelin J. Cellularity of bovine adipose tissues: developmental changes from 15 to 65 percent mature weight. J Lipid Res 1981; 22: 452-457.

29. Cianzio DS, Topel DG, Whitehurst GB et al. Adipose tissue growth and cellularity: changes in bovine adipocyte size and number. J Anim Sci 1985; 60: 970-976.

30. Poulos SP, Hausman DB and Hausman GJ. The development and endocrine function of adipose tissue. Mol Cell Endocrinol 2010; 323: 20-34.

31. Virtue $S$ and Vidal-Puig A. It's not how fat you are, it's what you do with it that counts. PLoS Biol 2008; 6: e237.

32. Mukesh M, Bionaz M, Graugnard DE et al. Adipose tissue depots of Holstein cows are immune responsive: inflammatory gene expression in vitro. Domest Anim Endocrin 2010; 38: 168-178.

33. Wan R, Du J, Ren L and Meng Q. Selective adipogenic effects of propionate in bovine intramuscular and subcutaneous preadipocytes. Meat Sci 2009; 82: 372-378.

34. Houseknecht KL, Baile CA, Matteri RL et al. The biology of leptin: a review. J Anim Sci 1998; 76: 1405-1420.

35. Chilliard Y, Delavaud C and Bonnet M. Leptin expression in ruminants: nutritional and physiological regulations in relation with energy metabolism. Domest Anim Endocrin 2005; 29: 3-22.

36. Delavaud C, Ferlay A, Faulconnier $Y$ et al. Plasma leptin concentration in adult cattle: effects of breed, adiposity, feeding level, and meal intake. J Anim Sci 2002; 80: 1317-1328.

37. Higashiyama $\mathrm{Y}, \mathrm{Abe} \mathrm{H}, \mathrm{Hayashi} \mathrm{M}$ et al. The comparison of plasma level and mRNA expression of leptin from Japanese Black steers and Holstein steers. Livest Prod Sci 2003; 81: 247-255.

38. Wang $\mathrm{P}$, Mariman E, Renes J et al. The secretory function of adipocytes in the physiology of white adipose tissue. J Cell Physiol 2008; 216: 3-13.

39. Ronti T, Lupattelli $G$ and Mannarino E. The endocrine function of adipose tissue: an update. Clin Endocrinol 2006; 64: 335-365.

40. Alzón M, Mendizabal JA, Arana A et al. Adipocyte cellularity in different adipose depots in bulls of seven Spanish breeds slaughtered at two body weights. Animal 2007; 1: 261-267.
41. Mendizabal JA, Albertí P, Eguinoa P et al. Adipocyte size and lipogenic enzyme activities in different adipose tissue depots in steers of local Spanish breeds. Anim Sci 1999; 69: 115-121.

42. Pike BV and Roberts CJ. Size and lipolytic capacity of bovine adipocytes from subcutaneous and internal adipose tissue. Vet Res Commun 1984; 8: 61-64.

43. Palmquist DL, Lock AL, Shingfield KJ et al. Biosynthesis of conjugated linoleic acid in ruminants and humans. Adv Food Nutr Res 2005; 50: 179-217.

44. Bessa RJB, Alves SP, Jerónimo E et al. Effect of lipid supplements on ruminal biohydrogenation intermediates and muscle fatty acids in lambs. Eur J Lipid Sci Technol 2007; 109: 868-878.

45. O'Sullivan A, O'Sullivan K, Galvin K et al. Grass silage versus maize silage effects on retail packaged beef quality. J Anim Sci 2002; 80: 1556-1563.

46. Shen X, Nuernberg K, Nuernberg G et al. Vaccenic acid and cis-9, trans-11 CLA in the rumen and different tissues of pasture- and concentrate-fed beef cattle. Lipids 2007; 42: 1093-1103.

47. Dance LJE, Matthews KR and Doran O. Effect of breed on fatty acid composition and stearoyl-CoA desaturase protein expression in the Semimembranosus muscle and subcutaneous adipose tissue of cattle. Livest Sci 2009; 125: 291-297.

48. Gotoh T, Albrecht E, Teuscher F et al. Differences in muscle and accretion in Japanese Black and European cattle. Meat Sci 2009; 82: 300-308.

49. Garcia MR, Amstalden M, Morrison CD et al. Age at puberty, total fat and conjugated linoleic acid content of carcass, and circulating metabolic hormones in beef heifers fed a diet high in linoleic acid beginning at four months of age. J Anim Sci 2003; 81: 261-268.

50. Fritsche J, Fritsche S, Solomon MB et al. Quantitative determination of conjugated linoleic acid isomers in beef fat. Eur J Lipid Sci Technol 2000; 102: 667-672.

51. Eguinoa P, Brocklehurst S, Arana A et al. Lipogenic enzyme activities in different adipose depots of Pirenaican and Holstein bulls and heifers taking into account adipocyte size. J Anim Sci 2003; 81: 432-440.

52. Sieberta BD, Pitchford WS, Kruka ZA et al. Differences in $\Delta 9$ desaturase activity between Jersey- and Limousin-sired cattle. Lipids 2003; 38: 539-543. 